\title{
Nuisances sonores des travaux de fondations : mesures et prévention
}

\section{F. ROCHER-LACOSTE}

Universite Paris Est

Laboratoine centre? des ponts et chaussées Division Mecanicue des sols, des roches et de géologie de l'ingénieur 58, bol Lefebvre 75015 Paris frederic rocher-lacoste@cpe.ti
Les chantiers tłe construction (bâtiments, ouvrages d'art, travaux routiers et ferroviaires...) occupent une place très importante dans l'activité économique nationale et internationale. El pourtant, ces chantiers créent, dans leur voisinage, des niveaux sonores supérieurs à $80 \mathrm{~dB}(\mathrm{~A})$. Il n"est donc pas étonnant que le bruit occasionne par les chantiers, en particulier en milieu urbain, soit l'une des ruisances les plus fréquerment citées par les riverains.

A partir d'études expérimentales en vraie grandeur de l'ímpact sonore et plus particulièrement d'une analyse des brutts engendres par le fonçage des pieux avec un vibrateur, un itarteau ou un trépideur, cet arlicle tente de mieux faire comprendre les phénomènes acoustiques liés à la technique du vibrofonçage et de proposer des solutions pour en diminuer les nuisances.

Mots-clés : bruit de chantier, vibrofonçage, battage, acoustique.

\section{Noise of foundation works: measures and prevention}

noise is one of the most frequemily cited nuisances.

From full-scale experimental studies of the sound generated and especially an analysis of noise caused by pile driving with a vibrator. a hydraulic hammer or aneumatic hammer, this article attempts to understand phenomena related to vibratory driving and to propose solutions to reduce nuisances.

Key words: construction noise, vibratory driving. impact driving, acoustics. 


\section{Introduction}

Contrairement à beaucoup d'autres problèmes relatifs à l'environnement, la pollution par le brutt continue à se développer et génère un nonbre croissant de plaintes de la part des personnes qui y sont exposées. La croissance des nuisances sonores est dommageable parce qu'elle a des effets négatifs sur" la santé à la fois directs et cumulés. Elle affecte toutes les générations, et a des implications socioculturelles, physiques et économígues.

La perception des vibrations associée au brutt ambiant peut augmenter l'effet de muisance sur ce que ressent le riverain. Sur un chantiel de vibrofonçage, une grande partie des bruits est générée par le matérè auxiliatre: mâts de guidage, chaines, élingues. groupes lydrauliques, guides métalliques, moteurs des engins de levage... Ce niveau sonore peut être diminué par des movens assez simples : utilisation de palplanches pincées, de lubrifiant dans les griffes libres, de plaques de frottement non métaliigues sur les guides (bois, matières synthétiques...) de groupes insonorisés, isolation acoustique des élingues, câbles et chaines de sécurité (enrobage par matériau isolant : mousse, caoutchouc...).

En plus du bruit sourd du battage, les moutons diesels, à la différence des marteaux hydrauliques, rejettent dans l'atmosphère des fumées noires ainsi qu'une fine pluie d’huile suivant l'âge des matértels et la qualité de leur entretien.

On s'intéresse ici aux bruits de chantier et plus particulièrement aux ondes sonores qui se propagent dans l'air et qui sont engendrées par le fonçage des pieux (métalliques et en béton armé) avec un vibrateur, an marteau ou un trépideur. Pour ce faire. une étude expérimentale en vrale grandeur de l'impact acoustique a été réalisée sur cing sites Verberie, Vesoul, Frejus, Monchy Saint-Éloi, Limelettel.

\section{Le bruit et sa perception}

Le bruit dans l’environnement est défini comme le bruit émis simultanément par l’ensemble des sour ces sonores. Les sources principales de bruit dans l'environnement incluent les trafics aérien, routier et ferroviaine, les industries, les chantiers de construction et les travaux publics, et le voisinage (tondeuses, cris, animaux tamiliers...). Les sources principales de bruit à l'intérieur des bâtiments d'habitation ou de bureaux sont les systèmes de ventilation, les machines de bureau, les appareils ménagers et le voisinage lla musique, la voix, bes pas...j.

Physiquement, il n'y a aucune distinction entre le son et le bruit. Le son est une perception sensorielle et la configuration complexe des ondes sonores est désignée par les termes de : bruit, musiçue, chant, cri... Le bruit quant à lui, est défini comme un son indésirable (Berglund et al., 1999).

La plupart des bruits de l'envirannement peuvent être décrits par plusieurs mesures simples. Les mesures qui quantifient le bruit prennent en compte le contenu fréquentiel des bruits, les niveaux de pression acoustique globaux ainsi que leurs variations dans le temps.
La pression acousticne due aux vibrations d"un bruit est I'amplitude de la variation de la pression autour de la pression atmosphérique moyenne. Le niveau de pression acoustique est un rapport de deux puissances. Il est donné en décibels par la formule:

$$
\left.L_{p}=10 \log \mid \frac{p^{2}}{p_{0}^{2}}\right\rceil
$$

où :

pest la pression acoustique efficace en pascals ;

$p_{1}$ est la pression acoustique de référence qui est égale à $20 \mu \mathrm{Pa}$ et qui correspond approximativement à la plus petite pression acoustique que l'oreille peut percevoir.

Lintervalle des niveaux acoustiques que les auditeurs humains peuvent détecter est très large... Par exemple, une augmentation de $3 \mathrm{~dB}(\mathrm{~A})$ correspond au doublement de la puissance sonore. En conséquence, ces niveaux ne peuvent pas être ajoutés ou ramenés à une moyenne arithmétique. La figure 1 présente une échelle simplifiée de différents types de bruit exprimés en décibel.

Dans l'Union européenne, environ $40 \%$ de la population est exposée au bruit du trafic routier, ce qui équivaut a un nivea de pression acoustique excédant 55 $\mathrm{dB}(\mathrm{A})$ pendant la journée, et $20 \%$ sont exposés à des niveaux excédant 65 dB(A). Lorsque tous les bruits de transport sont réunis, on estime que plus de la moitié des citoyens de l'Union européenne vit dans des zones qui ne leur assurent pas un minimum de confort acoustique. Pendant la ruit, plus de $30 \%$ de la population sont exposés à des niveaux de pression acoustique excédant $55 \mathrm{~dB}(\mathrm{~A})$, ce qui perturbe leur sommeil (Fig. 2). La nuisance sonore est également très importante dans les villes des pays en voie de développement. Elle est due principalement au trafic routier et dans les zones de trafic routier intense, les niveaux de pression acoustique pendant 24 heures peuvent atteindre $75-80 \mathrm{~dB}(\mathrm{~A})$.

Les bruits de chantier ne sont pas des bruits permanents (Fig. 3) comme le bruit de trafic mais ils peuvent atteindre ponctuellement, pendant la durée des travaux des niveaux sonores de 80 à 100 dB(A). On observe qu'un bruit impulsionnel est plus péniblement ressenti qu'un brujt régulier de même niveau sonore. De ce fait, un chantier de vibrofonçage sera perçu comme moins agressif qu'un chantier de battage.

Les normes et circulaire de référence françaises pour la caractértsation et la mesure des bruits sont:

- NF S31-110 - Caractérisation et mesurage des bruits de I'environnement - Grandeurs fondamentales et methodes générales d'évaluation;

- NF S31-010 - Caractérisation et mesurage des bruits de l'environnement - Méthodes particulières de mesurage :

- circulaire no 97-110 du 12/12/1997 relative à la prise en compte du bruit dans la construction de routes nouvelles ou l'anénagement de routes existantes du réseau national:

- NF EN ISO 3744 - Détermination des niveaux de puissance acoustique émis par les sources de bruit à partir de la pression acoustique ;

- NF EN 996/A2 - Prescriptions de sécurité. Matériel de battage. 


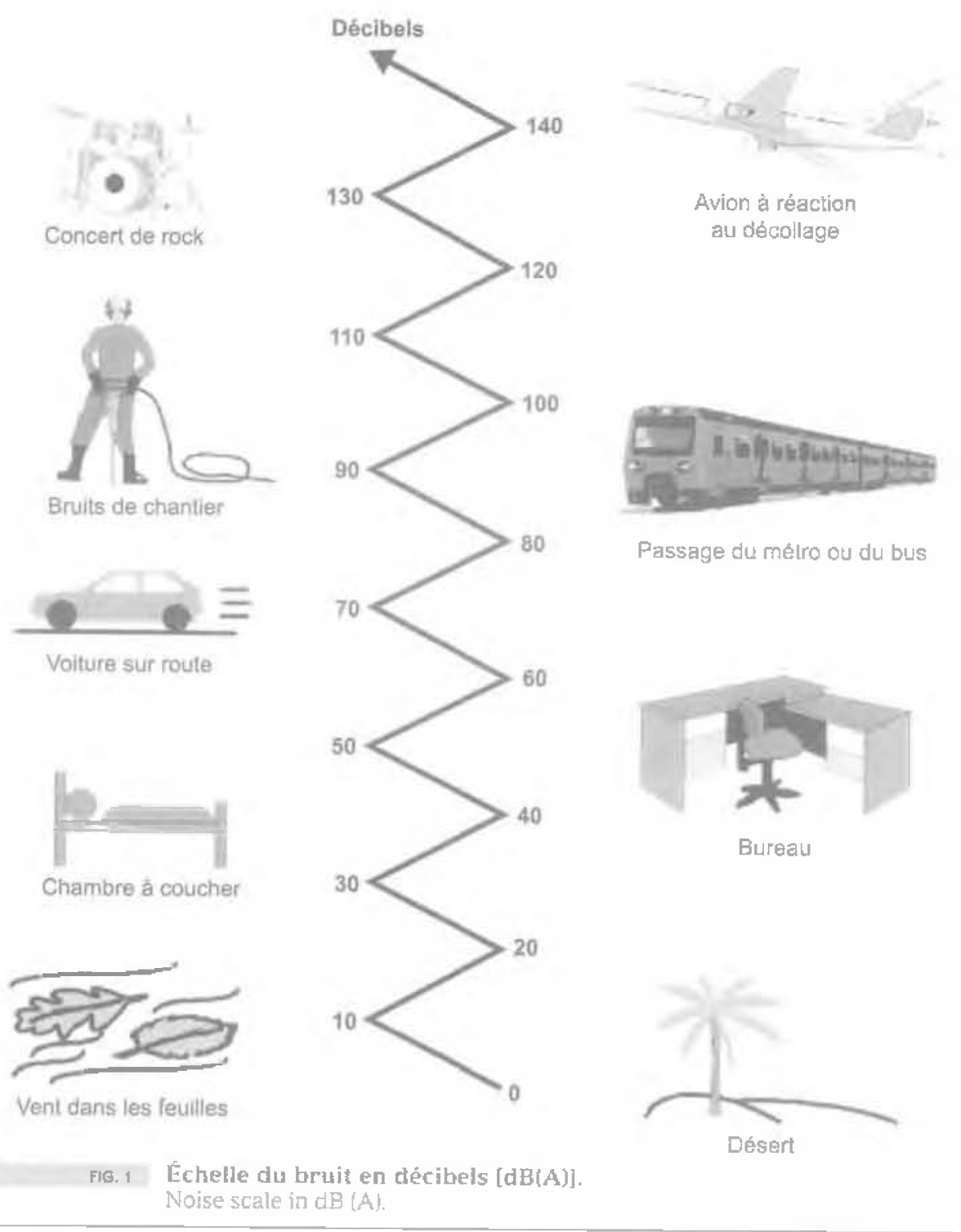

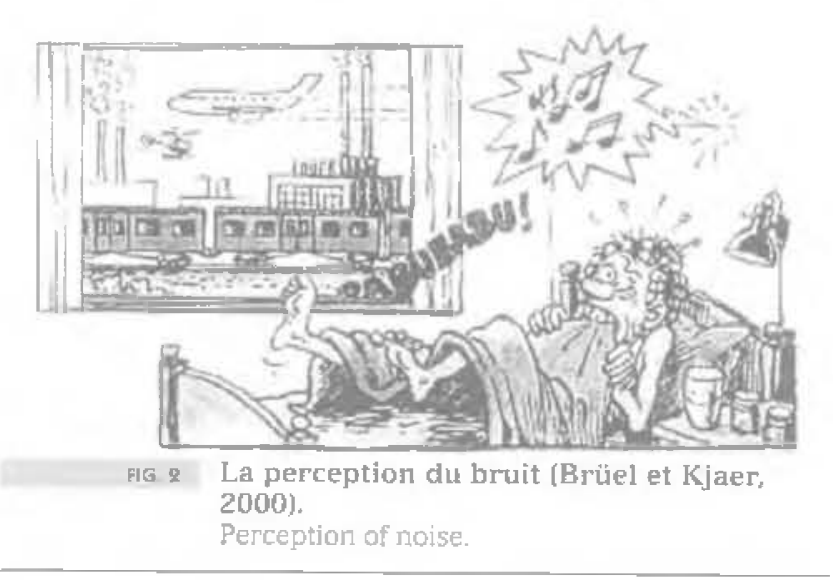

3

\section{Le bruit du chantier de vibrofonçage}

Le ressenti des bruits générés par les chantiers est lié à un sentiment de nuisances. Un chantier pour être furtif doit être réalisé sans gêner les riverains; c'est dans cette problématique que s'est inscrite cette étude sur les bruits de chantier dus au fonçage par vibrage des pieux métaliques ou en béton armé préfabriqué.

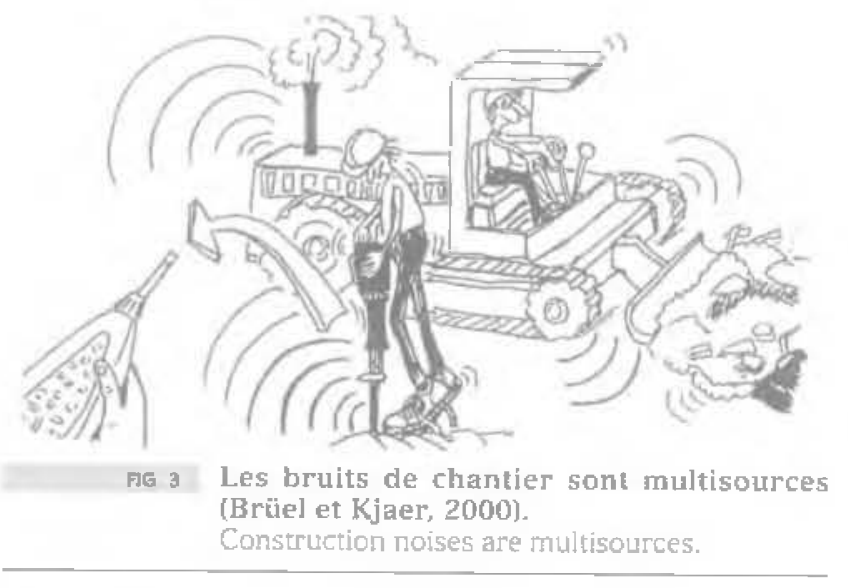

\section{Niveau de puissance acoustique}

On caractérise les engins de chantier par leur puissance acoustique. Elle s'exprime en décibels. La connaissance de cette valeur pernet de déterminer le niveau sonore d"un engin ä une distance donnée. La méthode de calcul proposée par Mériel et Bonhomme (1997) sépare le cas des sounces fuxes des sources mobt- 
taBLsad I Valeurs relevées par famille d'engins ou de matériels (Mériel et Bonhomme, 1997). Values mesured by machine or equipment families.

\begin{tabular}{|c|c|c|c|c|c|}
\hline$N^{0}$ & Nom de l'engin & Nombre d"engins & Lwa mini $d B(A)$ & Lwa maki dB(A) & Lwa moyen dB(A) \\
\hline 1 & Arroseur & 1 & 120 & 120 & 120 \\
\hline 2 & Autograde & 1 & 110 & 110 & 110 \\
\hline 3 & Balayeuse & 1 & 105 & 105 & 105 \\
\hline 4 & Bétonnièré portée & 3 & 91 & 98 & 96 \\
\hline 5 & Bouteur & 20 & 102 & 118 & 111 \\
\hline 6 & Camion & 9 & 95 & 109 & 106 \\
\hline 7 & Camion à peinture & 1 & 907 & 107 & 107 \\
\hline 8 & Centrale à blanc & 2 & 106 & 113 & 109 \\
\hline 9 & Chargeuse sur chenilles & 2 & 104 & 106 & 105 \\
\hline 10 & Chargeuse sur pneumatiques & 20 & 100 & 117 & 110 \\
\hline 11 & Chargeuse-pelletewse & 12 & 99 & 109 & 105 \\
\hline 12 & Chariot élévatelir & 2 & 98 & 102 & 100 \\
\hline 13 & Citeme avec motopompe & 1 & 103 & 103 & 103 \\
\hline 14 & Compactew à pied darneur & 2 & 115 & 116 & tit5 \\
\hline 15 & Compacteur monocylindre & 16 & 100 & 112 & 104 \\
\hline 16 & Compacteur sur preumatiques & 10 & 99 & 114 & 103 \\
\hline 17 & Compacteur statique & 1 & 101 & 101 & 101 \\
\hline 18 & Compacteur vibrant & 18 & 100 & 115 & 106 \\
\hline 19 & Compresseur (mono) & 5 & 90 & $11 \overline{7}$ & 106 \\
\hline 20 & Cancasseur & 1 & 117 & 117 & 117 \\
\hline 21 & Débroussailleuse & 2 & 101 & 100 & 103 \\
\hline 22 & Décapeuse automotrice & 6 & 108 & 118 & 111 \\
\hline 23 & Drague suceuse & 1 & 119 & 119 & 119 \\
\hline 24 & Excavateur & 1 & 110 & $\$ 10$ & 110 \\
\hline 25 & Finisseur & 8 & 107 & 113 & 109 \\
\hline 26 & Foreuse & 16 & 101 & 126 & 114 \\
\hline 27 & Gravillonneur & 3 & 98 & 102 & 98 \\
\hline 28 & Groupe de scudage & 1 & 110 & 110 & 110 \\
\hline 29 & Groupe électrogène & 10 & 89 & 116 & 109 \\
\hline 30 & Grue & $B$ & 94 & 114 & 107 \\
\hline 31 & Machine à cariveaux & 1 & 111 & 111 & 111 \\
\hline 32 & Marteau-piqueur & 6 & 112 & 121 & 116 \\
\hline 33 & Mouton - Marteau - Vibrateur & 20 & 104 & 132 & 158 \\
\hline 34 & Niveleuse automotrice & 18 & 94 & 113 & 105 \\
\hline 35 & Pelle sur chenilles < $100 \mathrm{~kW}$ & 14 & 103 & 111 & 107 \\
\hline 36 & Pelle sur chenilles > $100 \mathrm{~kW}$ & 18 & 102 & 116 & 100 \\
\hline 37 & Pelle sur pneumatiques & 14 & 102 & 112 & 106 \\
\hline 38 & Pelle avec brise-rocke & 8 & 114 & 123 & 118 \\
\hline 39 & Perforatrice & 1 & 121 & 121 & 121 \\
\hline 40 & Pilonneuse & 1 & 107 & 107 & 107 \\
\hline 41 & Répandeuse & 3 & 95 & 100 & 98 \\
\hline 42 & Scie à essence & 3 & 112 & 117 & 114 \\
\hline 43 & Scie sur tracteur & 1 & 114 & 114 & 114 \\
\hline 44 & Tombereas automoteur & 17 & 100 & 113 & 108 \\
\hline 45 & Vibreur & 1 & 108 & 108 & 108 \\
\hline
\end{tabular}


les. Le fonçage par vibrage et battage est assimilable à une source fixe ou faiblement mobile. La mesure doit être réalisée à une distance d*au moins une fois la plus grande dimension de l'engin ou matériel de chantier, à $1,2 \mathrm{~m}$ au-dessus du sol et à plus de $3 \mathrm{~m}$ de toute surface réfléchissante. On obtient ainsi le niveau acoustique équivalent LAeqi par cycle de travaíl représentatif pour chaque point de mesure, i.

Dans le cas d'un sol réfléchissant. le niveau de puissance LWA est donné par :

$$
\mathrm{LWA}=\mathrm{LAEq}+20 \log \mathrm{d}+\mathrm{g}
$$

où d est la distance "source-point de mesure $w$ en mètres.

On calcule ensuite la valeur moyenne de LWA par:

$$
\operatorname{LwA}_{\text {moyen }}=10 \log \left[\frac{1}{N} \sum_{=1}^{N} 10^{0,14 n}\right]
$$

où N est le nombre de points de mesures.

\section{Études antérieures}

Il existe peu de publications ou de données comparatives traitant des bruits incluits par le fonçage par vibrage et par battage de pieux. C'est pourquoi nous détaillons ci-après deux publications marquantes concernant les nuisances des chantiers de travaux publics. Elles posent les premières bases sur lesquelles nos travaux se sont appuyés.

En 1997, Mériel et Bonhomme ont publié dans le Bulletin des laboratoires des ponts et chaussées une synthẻse sur le bruit émis par les chantiers en milieu urbain. Le tableau I permet de se faire une idé du bmult émis par différents engins de chantien, mais il ne permet pas de faire une comparaison entre le vibrofonçage et le battage, car ces deux méthodes sont regroupées sur la même ligne 33 intitulée a Mouton - Marteau - Vibrateur ". Il ne faut pas confondre cet item avec la ligne 45 a vibreur a gui traite du vibreur utilisé pour aider

En 2002. le Laboratore régional des ponts et chaussées de Blois a réalisé une étude acoustique (Boittin, 2002) sur Ia commune de La Riche (Indre-et-Loire) concernant un chantier SNCF ayant pour objectif de supprimer un passage à niveau. Des palplanches simples de type PU 20 de $8 \mathrm{~m}$ de long ont été vibrofoncées avec un vibrateur ICE 14RF et battues avec un marteau hydraulique IHC S35. Sur le chantier, à 26 m de la source (zone de fonçage des palplanchesl, il a été mesuré une différence de puissance acoustique entre le fonçage par battage et par vibrage de 5 dB(A). [Battage : LWA = $130 \mathrm{~dB}(\mathrm{~A})$, Vibrofonçage: LwA = $125 \mathrm{~dB}(\mathrm{~A})]$. Dans cette étude, le battage a été le plus bruyant des deux modes de mise en couvre observés.

\section{Études récentes}

\section{Introduction}

Le Projet national Vibrofoncage (Guide technique Vibrofoncage. 2006) n'évoguant qué succinctement la question du bruit généré et ne présentant aucune mesure, nous avons effectué des mesures acoustiques pendant les opérations de fonçage par wibrage dans le cadre de chantiers expérimentaux (Verberie et Limelettel et de chantiers réels (Vesoul. Fréjus et Monchy Saint-Éloi).

Suivant les sites, des mesures ont été affectuées en fonction de l'enfoncement soit en un point fixe, soit k différentes distances de l'élément foncé pour obtenir des courbes moyennes d'atténuation. Pour essayer de déterminer l'augmentation de pression acoustique due au vibrofonçage (ensemble vibrateur/pieu), des mesures ont aussi été réalisées avant la mise en service du chanțer.

Deux types de sonomètre ont été utilisés pour l'acquisition acoustique pour l'ensemble des sites de mesures:

- sonomètre ACLAN, type SGD 80;

- sonometre EXTECH instruments, model 407764.

\section{Site de Verberie}

Une étude de vibrations menée par le LCPC et ICE. France lors de la mise en ouvre de palplanches de type PU25 a été réalisée sur le site de Verberie en mars.

tamerau II Mesures acoustiques ponctuelles sur le site de Verberie (meme point de mesure).

Acoustic measures or the Verberie site isame point of measuretheni.'.

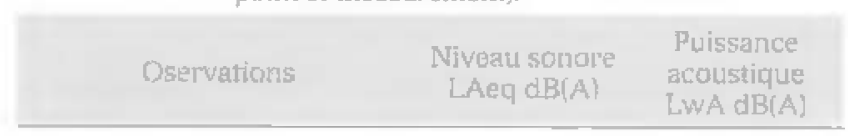

Bruits d'ateller a 50 m du poht de mesure

60 à 70

Bruit de la grue seule à 10 a du point de mesture

85 113

Brubt du vibrateur seul à $10 \mathrm{~m}$ du point de mesure

\begin{tabular}{|c|c|c|c|c|}
\hline TABLEAU III & $\begin{array}{l}\text { Mesures acou } \\
\text { Acoustic meas: }\end{array}$ & $\begin{array}{l}\text { lifférents } \\
\text { ent places }\end{array}$ & $\begin{array}{l}\text { ents sur le } \\
\text { eria site. }\end{array}$ & de Verberie. \\
\hline \multirow{2}{*}{ Palpianche } & & sonore LAE & & \multirow{2}{*}{ Otoserwations } \\
\hline & à $5 \mathrm{~m}$ & a $10 \mathrm{~m}$ & à $15 \mathrm{IJ1}$ & \\
\hline 1 & $\begin{array}{l}\text { Min : } 82 \\
\text { Max : } 87\end{array}$ & $\begin{array}{l}\text { Min: } 74 \\
\text { Max: } 83\end{array}$ & & Sans brult parasite \\
\hline 2 & & $\begin{array}{l}\text { Min }=85 \\
\text { Max }=98\end{array}$ & $\begin{array}{l}\text { Mir : } 85 \\
\text { Max : } 97\end{array}$ & Nettoyage du parc au karcher \\
\hline 3 & & $\begin{array}{l}\text { Min }=84 \\
\text { Max: } 92\end{array}$ & $\begin{array}{l}\text { Min: } 73 \\
\text { Max : } 82\end{array}$ & Bruits dvers sur le parc \\
\hline
\end{tabular}

116 
2006. Les palplanches ont été mises en œuvre avec un vibrateur ICE 216, dans un champ mitoyen du dépôt de matériels de l"entteprise ICE-France. L'idée de mesurer le niveau sonore dû̉ au fonçage par vibrage s'est tmposée au cours de cette expérience.

Des mesures ont été réalisées avant le début des essais pour avoir une idée générale du bruit ambiant sur le site. La proximité de l'atelier de maintenance des vibrateurs parasite fortement les mesures avec un bruit de fond êtevé et variable. Le tableau II montre qu'il y a peu de différence cle niveau sonore entre le vibrateur avec son groupe hydrauliąue et la grue seule qui est ancienne et très bruyante.

Au total sur ce site, on a réalisé trois fonçages par vibrage de la mềme palplanche PU25. Des mesures acoustiques ont été collectées en même temps que des mesures de vitesses particulaires au niveau des points de mesures des géophones, respectivement à 5,10 et $15 \mathrm{~m}$ de la palplanche. Le tableau II] regroupe ces mesures. La présence d'un atelier bruyant n'a pas permis d'exploiter les mesures faites trop masquées par les bruits parasites.

\section{Site de Vesoul}

Deux campagnes de mesures pendant la mise en cuvre de pieux $\mathrm{H}$ et de palplanches ont été entreprises les vendredi 21 avril et mardi 23 má 2006 à Vesoul sur le chantier du groupement Pertuy/Vilault pour réaliser un passage inférieur sous les voies SNCF, entre un jardin public et une place, à proximité de la gare SNCF. pendant les phases de fonçage, des pieux HEB 300 ont été mis en cuvre avec un vibrofonceuria PTC $20 H F 3$ et avec un trépideur Menck SB120, et des paires de palplanches soudées de type PU 16 ont été foncées avec un vibrateur ICE 416 .

Le tableau IV regroupe l'ensemble des mesures ponctuelles collectées pendant les deux campagnes de mesures sur ce site, pour des sources differentes et à des distances variables ainsi que les valeurs calculées de la puissance acoustique (éq. 2). L'analyse des données montre que :

- le bruit ambiant de la ville est compris entre 50 et $60 \mathrm{~dB}(\mathrm{~A})$;

- l'utilisation du trépideur augmente le njveau sonore de $20 \mathrm{~dB}(\mathrm{~A})$ :

- le groupe hydraulique utilisé le 21 avril est plus bruyant que celui utilisé le 23 mai

- le fonçage avec un trépideur est globalement plus bruyant que le fonçage par vibrage. La puissance acoustique du trépideur ne change pas en fonction de l'ếlö́ment foncé.

Le tableau V rassemble les mesures qui ont été collectées pendant le fonçge à différentes distances de la source $(5,10,15$ et $20 \mathrm{~m})$ pour !es deux campagnes d'essai. Le 21 avril l'entreprise a essayé de foncer des pieux HEB 300, mais le vibrateur se mettait en sécurité

\begin{tabular}{|c|c|c|c|}
\hline Date et heure & Dbservations & $\begin{array}{l}\text { Niveau sonore } \\
\text { LAeq dB!A! }\end{array}$ & $\begin{array}{l}\text { Puissance acoustique } \\
\text { LwA tBi A] }\end{array}$ \\
\hline $\begin{array}{l}21 \text { avril } 2006 \\
7 h 15\end{array}$ & Bruits de la ville & $50-60$ & \\
\hline $\begin{array}{l}23 \text { mai } 20065 \\
9 \text { h } 00\end{array}$ & Bruits de la ville & $53-58$ & \\
\hline $\begin{array}{l}21 \text { avrli } 2006 \\
8 \text { h } 10\end{array}$ & $\begin{array}{l}\text { Groupe + trépideur lors de l'enfoncement } \\
\text { de palplanches : } 36 \mathrm{~m} \text { du point de mesure }\end{array}$ & 90 & 129 \\
\hline $\begin{array}{l}21 \text { avril } 2006 \\
8 \text { th } 25\end{array}$ & Groupe seul : 36 m du point de mesure & 70 & 109 \\
\hline $\begin{array}{l}23 \operatorname{mal} 2006 \\
9 \mathrm{~h} 20\end{array}$ & Groupe seul : $5 \mathrm{~m}$ du point de mesure & 63 & 85 \\
\hline $\begin{array}{l}21 \text { avril } 2006 \\
101700\end{array}$ & 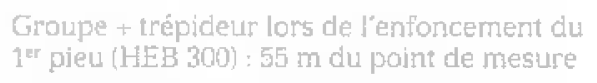 & 85 & 128 \\
\hline $\begin{array}{l}23 \text { mai } 2006 \\
\text { 9t } 30\end{array}$ & $\begin{array}{l}\text { Groupe + vibrateur lors de l'enfoncement } \\
\text { de palplanches ä } 30 \mathrm{~m} \text { du point de meswe }\end{array}$ & 83 & 121 \\
\hline
\end{tabular}

TAgleau Y Mesures acousticues a différentes distances de la source sur le site de Vesoul. Acoustic measures at different places from the solurce on the Vesoul site.

\begin{tabular}{|c|c|c|c|c|c|}
\hline Elf́ment & Mode lientoncement & & Nivea & Aeq dBIA & \\
\hline Livitent & 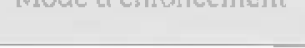 & 当当第 & aे 10 ז & à 15 m & à $20 \mathrm{~m}$ \\
\hline Pien $n^{\square}$ & Vibrateur & & Max : 98 & & 75 \\
\hline & Trepideur & $\begin{array}{l}\text { Min : } 95 \\
M a x: 116\end{array}$ & $\begin{array}{c}\text { Miг] }=90 \\
\text { Max }=112,6\end{array}$ & & 92 \\
\hline Pleu $\pi^{\circ} 2$ & Trépideur & Max 114 & 95 & 93 & 90 \\
\hline PPL $\pi^{\circ} 1$ & Vibratew" & 89 & 98 & 96 & 83 \\
\hline PPL $\cap^{\circ} 2$ & Vibrateus & 88 & 86 & 84 & 82 \\
\hline $\mathrm{PPL} \pi^{a} 3$ & Vibrateur & 95 & 94 & 89 & 88 \\
\hline PPL $\pi^{\circ} 4$ & Vibrateur & & $80-93$ & & \\
\hline Moyenne vibrateur [PPL] & & 91 & 89 & 86 & 84 \\
\hline Moyenne urépideur [Pleu] & & 106 & 98 & 93 & 91 \\
\hline
\end{tabular}




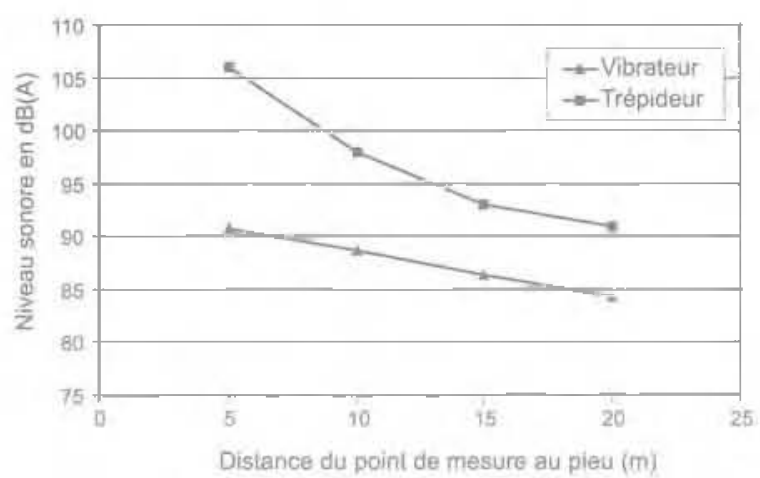

EFG. 4 Courbes d"attenuation à partir des moyennes (vibrateur et trépideur) du niveau sonore en décibels en fonction de la distance par rapport à la source.

Reduction of noise level in dB(A) from mean values [vibrator and pneumatic hammert according to the distance of the source.

après guelcues secondes d'utilisation. Il n'a pas été possible de mener à bien un fonçage par vibrage avec cet équipement. Avec trépideur, le fonçage était très laborieux et très bruyant. Le 23 mai, quatre fonçages par vibrage de palplanche ont été réalisés.

La figure 4 montre une intensité sonore nettement supérieure du trépideur, intensité qui tend cependant à s'atténuer plus rapidement que celle du vibrateur. Cette interprétation peut être sujet à crịique car les éléments foncés avec ces deux méthodes n'étaient pas les mêmes : pieu HEB 300 avec le trépideur et palplanche PU 16 avec le vibrateus.

\section{Site de Fréjus}

Conrre pour le chantier de Vesoul, il a été possible, en juillet 2006 à Fréjus, de fáre des mesures pendant la mise en oevve par vibrage de palplanches. Ces mesures ont été effectuées, avec le soutien de la SNCF, sur le chantier d'aménagement du carrefour RN7/RD4, entre une zone industrielle et les voies ferroviaires d'accès à la gare de Fréjus. Pour réaliser le soutènement d"un passage inférieur, des palplanches simples de type L603 de 8 à $10 \mathrm{~m}$ de Jongueur ont été mises en ouvre avec un vibrofonceur PTC 30 HF3A et un guide de battage métaliique.

Les valeurs de niveau sonore du chantier (Tableau VI) montrent que le bruit dù à la circulation routière esit un facteur prépondérant au sein du bruit général. Cette constatation n'est pas surprenante puisque le chantier était situé entre deux voies de circulation.

Les mesures collectées (Tableau VIi) pendant le fonçage, à des distances différentes du point de fonçage $(10,20,40$ el $80 \mathrm{~m})$, pour six des onze palplanches mises en ceuve, montrent un net accroissement du niveau sonore d"environ $15 \mathrm{~dB}(\mathrm{~A})$ par rapport au bruit de circulation. Aucune mesure acoustique n'a été effectuée Sur les cing attres palplanches.

Pour I'ensemble des six essais. I puissance acous tique moyenne LwA now (éq. 2) est égale à 124 dBIA. pour le vibrateur PTC 30HF3A et la palplanche L603.

La figure 5 représente la courbe d'atténuation de la moverne des riveaux sonores des six fonçaces de palplanches et des niveaux sonores calculés à partir de la

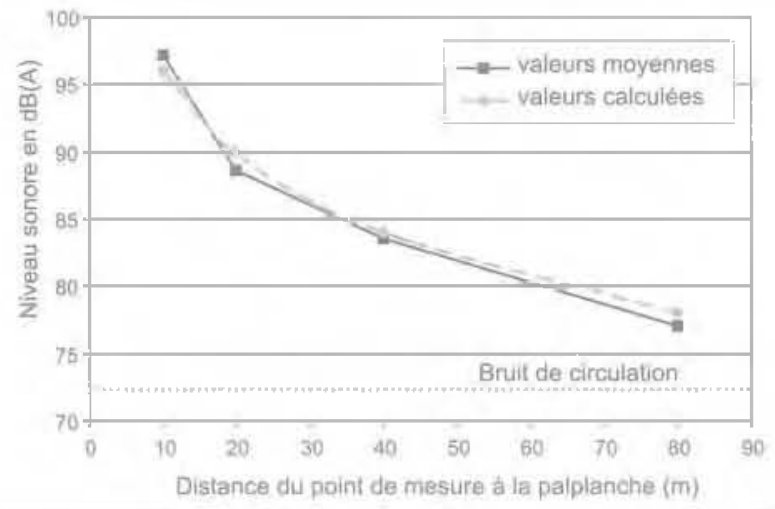

ms. 3 Diminution du niveau sonore en fonction de la distance par rapport a la source.

Reduction of noise level according to the distance from the source.

\begin{tabular}{|c|c|c|}
\hline TABLEAU W & $\begin{array}{l}\text { S acoustiques à differents } \\
\text { measures at different places }\end{array}$ & $\begin{array}{l}\text { acements sur le site de Fréjus. } \\
\text { Eréjus site. }\end{array}$ \\
\hline Heure & Niveau sonore IAec dB:Al & Observations \\
\hline $8 \cap 45$ & 72 & Bruits de la circulation au point de référence [env à $60 \mathrm{~m}$ ] \\
\hline $8 h 4 \overline{5}$ & 63 & Bruit sans passage de voiture au point de référence (erv à $60 \mathrm{~m}$ ) \\
\hline $8 \mathrm{~h} 45$ & 67 & Bruit de la circulation + bruit du chantier (mini-peliel ä $40 \mathrm{~m}$ \\
\hline 10 h 00 & 70.3 & Brult de la grue et de la circulation au point de rêfêrence (env, $60 \mathrm{~m}$ ) \\
\hline $70 \mathrm{H} 00$ & 73.5 & Bruit de la grue et de la circulation à $10 \mathrm{~m}$ \\
\hline
\end{tabular}

zanteau vi Mesures acoustiques à diffểrentes distances par rapport au point de fonçage sur le site de Fréjus. Acoustic measures at different places according to the boritig point on the Frefus site.

\begin{tabular}{|c|c|c|c|c|c|}
\hline \multirow{2}{*}{ Elemęnt } & \multirow{2}{*}{ Mode d'entoncement } & \multicolumn{4}{|c|}{ Niveau satmone LAeq dBMA] } \\
\hline & & à $10 \mathrm{~m}$ & à $20 \mathrm{~m}$ & ה 40 & $80 \mathrm{~m}$ \\
\hline Ppl ñ I & Vibrateur & 97.5 & 87.7 & 82,4 & \\
\hline $\operatorname{Ppl~}^{\circ} 2$ & Vibrateur & 98,9 & 88.7 & 85,4 & 77,5 \\
\hline $\mathrm{Ppl} n^{2} 3$ & Vibrateur & & 91,6 & 87.9 & $76, \mathrm{~B}$ \\
\hline $\mathrm{Ppln} \mathrm{n}^{\mathrm{n}} 4$ & Vibrateur & & 86,1 & 82,3 & 76.9 \\
\hline Ppl $n^{0} 5$ & Vibrateur & 96,1 & 88,0 & 77.9 & \\
\hline Ppl n $n^{0} 6$ & Vibrateur & 96,2 & 92,5 & 85,5 & \\
\hline \multirow{2}{*}{\multicolumn{2}{|c|}{$\begin{array}{l}\text { Moyerne arithmétique des niveaux sonores mesurés } \\
\text { Nweau sonore calculé à partir de la puissance acoustique } \\
\text { [LwA moyen] }\end{array}$}} & 97 & 89 & 84 & 77 \\
\hline & & 96 & 90 & 84 & 78 \\
\hline
\end{tabular}


puissance acoustique (éq. 2) en fonction de la distance à la source. Les valeurs dites calculées et les valeurs mesurées sont voisines, ce qui valide l'éçuation 2.

De même, on peut estimer que le bruit de circulation est quasiment prépondérant au-delả de $80 \mathrm{~m}$.

\section{(19)}

\section{Site de Monchy Saint-Éloi}

Pour valider" les dimensionnements et la méthode de mise en cetwre des fondations de la future station d'épuration de Monchy, l'entreprise NGE a mis en ceuvre, en mai 2007 , avec un vibrateur ICE 416 des pieux en béton armé carrés de $500 \mathrm{~mm}$. Le chantier est situé entre l'ancienne station d'épuration, un cours d'eau el ure forêt. Pour pouvoir faire un essai comparatif de capacité portante, ur des pieux a été sur-battu de $15 \mathrm{~cm}$ avec un marteau hydraulique IHC SC50. Des mesures acoustiques ont éte faites pendant la mise en cuvre du płeu vibrofoncé puis sur-battu à $15 \mathrm{~m}$ de celui ci [Tableau VII). Suite à ces mesures il est possible de conclure dans ce cas précis que le fonçage par battage est environ $20 \mathrm{~dB}(\mathrm{~A})$ plus bruyant que le fonçage par vibrage, malgré l'installation d'un coussin d"amortissement en bois entre l'enclume (acier) et la tête de pieu (béton) pour éviter de casser cette dernière.

\section{Site de Limelette}

Du 23 au 25 octobre 2007, une mêne paire de palplanches $Z$. de 20 m de longueus, qui avait les griffes soudẻes, a èté foncée et extraite, neuf fois, par vibrage avec un vibrateur ICE 36RF-ts. Les essais ont téé réalisés sur un site dégagé du Centre Scientifique et Technique de la Construction à Limelette (Belgique). La puissance acoustique de la grue en mouvement, mesurée à $20 \mathrm{~m}$, est de $104 \mathrm{~dB}(\mathrm{~A})$.

Pendant chaque phase de fonçage, des mesures de niveau sonore ont été effectuées à $5,10,2030$ ou $40 \mathrm{~m}$ de distance de la source vibrante. Les mesures ont aussi été réalisées en fonction de la pénétration de 0 à $7 \mathrm{~m}$. Le tableau IX présente une synthèse de ces mesures ainsi que le calcul de la puissance acoustique moyenne (éq. 1 et 2) pour chacun des essais. La figure 6 montre sous forme de courbes la moyenne des niveaux sonores en fonction de la distance de la source vibrante.

Trois paramètres étaient susceptibles de varier d'un essai à l'autre : le nombre de pinces (une ou dewx) gui tienrent la palplanche, la fréquence et l'amplitude du vibrateur. En associant les essais deux par deux, ou trois par trois, il est possible de faire les observations suivantes.

\section{Essais A, i et $i^{\circ}$}

Pour ces essais, les trois parametres sont identiques : fréquence maximale de $37,5 \mathrm{~Hz}$, amplitude maximale de 3,8 à 4 mm et une seul pince. Notons que le Guide technique Vibrofonçage recommande d'utiliser deux pinces mais que les entrepreneurs ont tendance à n'en utiliser qu'une. Lessai A semble légèrement plus bruyant que les essais I et $I^{*}$, qui ont des valeurs de puissance acoustique similaires. Le fait que l'essai A soit le premier" essai du chantier expérimental [essai de faisabilité et cle calagel pourrait expliquer cette légère différence.

\begin{tabular}{|c|c|c|c|}
\hline \multirow[t]{2}{*}{ CHETEAU YII } & \multicolumn{3}{|c|}{$\begin{array}{l}\text { Mesures acoustiques a } 15 \mathrm{~m} \text { du point de fonçage sur le site de Monchy. } \\
\text { Acoustic measures at } 15 \text { m of the boring point on the Monchy site. }\end{array}$} \\
\hline & Observations & $\begin{array}{l}\text { Nivenu sumore } \\
\text { LAeq dB|Al }\end{array}$ & $\begin{array}{l}\text { Puissance acousticite } \\
\qquad W W A \text { dB(A) }\end{array}$ \\
\hline \multicolumn{2}{|c|}{ Niveau sonore initial (chantier à l'errết) } & 49 & \\
\hline \multicolumn{2}{|c|}{ Groupe seut } & 62 & 94 \\
\hline \multicolumn{2}{|c|}{ Grue au ralenti } & 66 & 98 \\
\hline \multicolumn{2}{|c|}{ Grue en action + groupe } & 87 & \\
\hline \multicolumn{2}{|c|}{ Grue seule en action } & 73 & 105 \\
\hline \multicolumn{2}{|c|}{ Gme + groupe + vibrateur : phase de vibrofonçage } & $\begin{array}{l}\operatorname{Min}: 76 \\
\operatorname{Max}: 80\end{array}$ & $\begin{array}{l}\operatorname{Min}: 108 \\
\operatorname{Max}: 112\end{array}$ \\
\hline \multicolumn{2}{|c|}{ Grue + groupe + marteal : phase de battage } & 98 & 130 \\
\hline
\end{tabular}

\begin{tabular}{|c|c|c|c|c|c|c|c|c|c|}
\hline \multirow{3}{*}{$\begin{array}{l}\text { Essai } \\
\text { \{ppl] }\end{array}$} & \multicolumn{9}{|c|}{$\begin{array}{l}\text { Synthese des differents essais. } \\
\text { Synthesis of different test. }\end{array}$} \\
\hline & \multirow{2}{*}{ Pinct } & \multirow{2}{*}{$\begin{array}{l}\text { Frequence } \\
\text { (Atz) }\end{array}$} & \multirow{2}{*}{$\begin{array}{l}\text { Amplitude } \\
\text { [mm] }\end{array}$} & \multicolumn{5}{|c|}{ Moyerine du niveati sonore LAeg dBiAj) } & \multirow{2}{*}{$\begin{array}{l}\text { Puissance acousticflit } \\
\qquad \text { LWA }_{\text {timy }} \mathrm{dB}|\mathrm{A}|\end{array}$} \\
\hline & & & & à $5 \mathrm{~m}$ & $10 \mathrm{~m}$ & â 20 m & an $30 \mathrm{~m}$ & 본 40 & \\
\hline A & sitnple & 37.5 & 4 & 101 & 99 & 93 & 87 & & 126 \\
\hline $\mathrm{B}$ & sitrple & 37,5 & 2 & 95 & 91 & 85 & 82 & & 119 \\
\hline C & sitiplie & 33 & 4.2 & 96 & 92 & 87 & & 82 & 121 \\
\hline D & sitiple & 28.5 & 4,1 & 94 & 89 & 82 & & $7 \overline{7}$ & 17 \\
\hline E & simple & 31,5 & 1.4 & 95 & 92 & 84 & 79 & & 118 \\
\hline$F$ & double & 33 & 2,6 & 93 & 88 & 84 & 83 & & 118 \\
\hline$G$ & double & 32,5 & 4,5 & 93 & 87 & 82 & 79 & & 116 \\
\hline $\mathrm{H}$ & double & 35.20 & 4,5 & 92 & 89 & 84 & 80 & & 117 \\
\hline 1 & simple & 37.5 & 4 & 99 & 96 & 50 & 85 & & 123 \\
\hline$\amalg^{\circ}$ & simple & 37,5 & 3,8 & 103 & 96 & 90 & 84 & & 124 \\
\hline
\end{tabular}




\section{Essais $C, D$ et !}

Pour ces essais, deux paramètres sont identiques ou equivalents : amplitude maximale de 4 à $4,2 \mathrm{~mm}$, une seule pince et la fréquence varie. On constate que plus la fréquence est élevée, plus la puissance acoustigue est forte $\left(\mathrm{LWA}_{5.5}=123>\mathrm{LWA}_{3,3}=121>\mathrm{LWA}_{2.5}=117\right)$.

\section{Essais Cet $G$}

Pour ces essais, deux paramètres sont identiques fréquence de 32,5 et $33 \mathrm{~Hz}$ amplitude maximale de 4,2 et $4.5 \mathrm{~mm}$ et le nombre de pince varie. On observe une différence de 5 dB $[$ Al. Le fonçage avec une pince simple est donc plus bruyant ( $L W A_{s}=121>L_{W A}=117$ ).

\section{Essais B et I}

Pour ces essais, deux paramètres sont identiques: fréquence maximale de $37,5 \mathrm{~Hz}$ et une seule pince et l'amplitude varje de 2 à $4 \mathrm{~mm}$. On observe une différence de $4 \mathrm{~dB}(\mathrm{~A})$. Le toncage avec une amplitude importante serait plus bruyant (LWA $\left.=123>\mathrm{LWA}_{\mathrm{f}}=119\right)$.

\section{Essais C et E}

Pour ces essais, delx parametres sont identiques fréquence de 31,5 à $33 \mathrm{~Hz}$ et une seule pince et l'amplì tude varie de 1,4 à $4,2 \mathrm{~mm}$. On observe une différence de $3 \mathrm{~dB}(\mathrm{~A})$. Le fonçage avec une amplitude importante

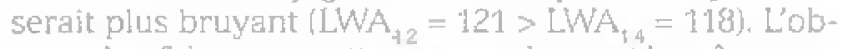
servation faite pour cette comparaison est la même que précédemment (essais B et I). Peut-on en déduire que, quelle que soit la fréquence, plus l'amplitude est élevée plus la puissance acoustique sera elevée?

\section{Essais F et G}

Pour ces essais, deux parametres sont identiques fréquence de 32,5 et $33 \mathrm{~Hz}$ et deux pinces et l'amplitude varie de 2,6 a $4,5 \mathrm{~mm}$. On observe une différence de 2 $\mathrm{dB}(\mathrm{A})$; cela est presque négligeable. On constate que le fonçage avec une amplitude faible serait légèrement plus bruyant $\left\{\mathrm{LWA}_{2, \mathrm{~h}}=118>\mathrm{LWA}_{4}=116\right)$. Cette observation n'est pas en concordance avec les comparaisons précédentes (essais C et E et essais B et I).

Ces essais mettent en évidence qu'll existerait une la relation entre la puissance mécanique et la puissance acoustique. En effet, la puissance mécanique serait, a priori, proportionnelle à l'amplitude de la force et à la fréquence qui sont appliguêes en tête de palplanche. Donc, jl n'est pas surprenant que la puissance acoustique augmente quand l'amplitude de la force et la fréquence augrmentent. Nous avons vérifié cela pour tous les essais sauf dans la comparaison F-G. Pour confirmer ou infirmer les observations faites, il faudrait réali-

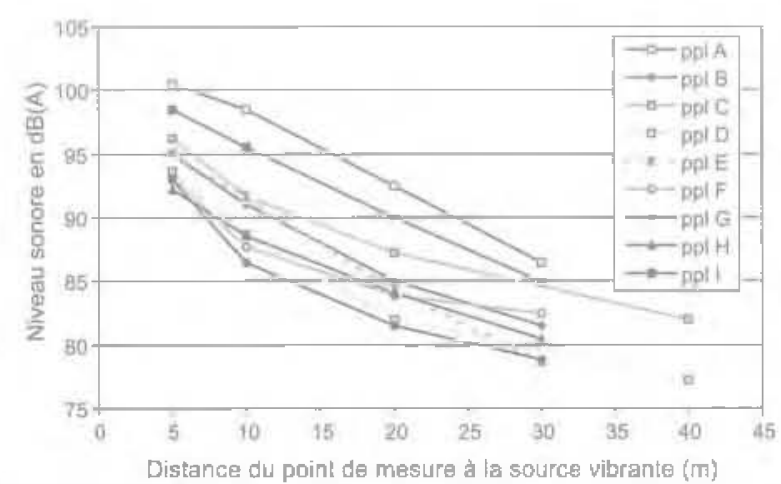

月6. Niveaux sonores moyens en fonction de la distance par rapport à la source.

Sound levels according to the distance from the source. ser d’autres thesures et constituer une banque de données acoustiques.

\section{4}

\section{Recommandations pour réduire les nuisances acoustiques}

Les travaux de battage ow/et vibrofonçage des pieux, palplanches et profilés métalliques ont la mauvaise réputation d'être bruyants et responsables de désagrément et nuisances dus aux vibrations du sol. Les riverains de ces chantiers ayant du mai à juger objectivement les effets des vibrations transmises dans le sol et dans l'air, considèrent souvent que ces vibrations sont à l'origine de désordres survenus sur leurs propriétés. Pour éviter des contentieux lors de l'exécution des travaux de battage ou de vibrofonçage, voire l'arrêt momentané du chantier, il est nécessaire. pour les travaux en zone urbaine et à proximité des habitations, de prendre des dispositions prévertives appropriées au site et en accord avec les nomes et les réglements en vigueur.

Les observations faites sur plusieurs chantiers de vibrofonçage montrent que ceux-ci sont nettement molns bruyants gue les chantiers gui utilisent des marteaux ou des trépideurs.

En plus du choix de la méthode de fonçage, le choix de la méthode de travail a une inftuence sur le type de nusisances produites : avec un mât de fonçage ou en pendulaire avec un guide de fonçage ou non. Par exemple, un guide en acier (Fig. 7) amplifie le bruit (contact acier/acier), ce qui amene ì privilếgler l'utilisation d'un guide à structure bois (Fig. 8).
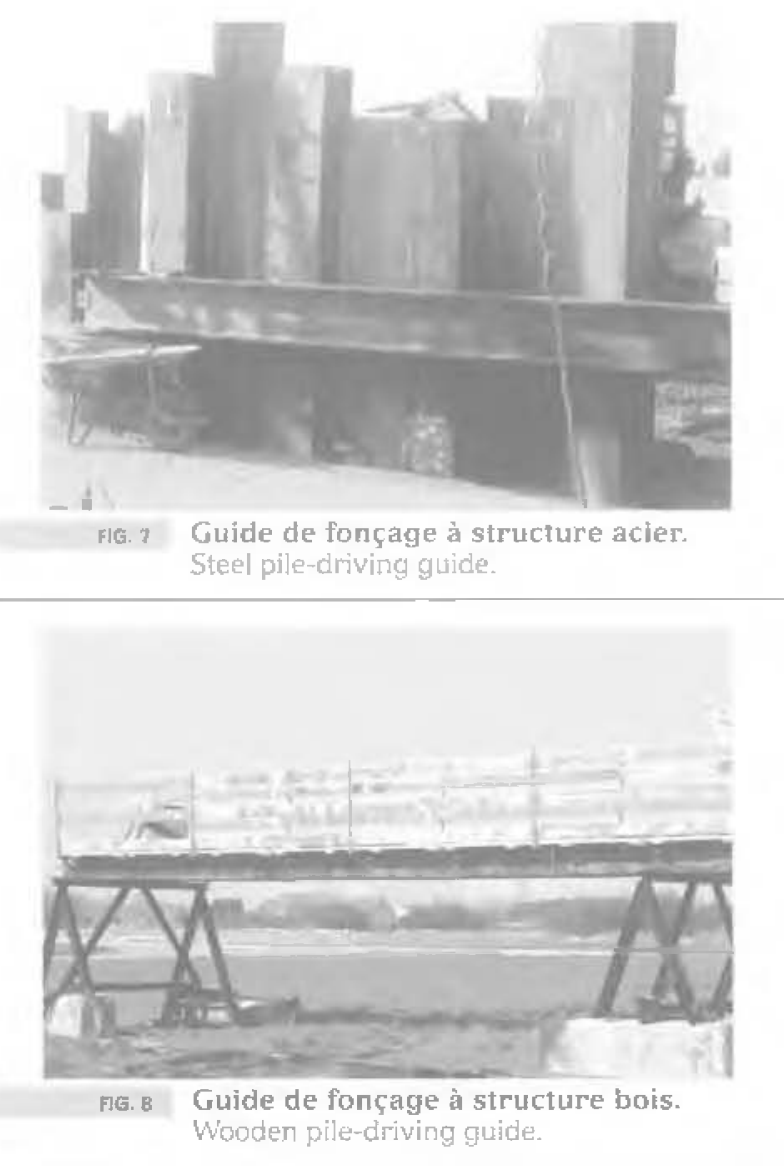
Pour canaliser le bruit, îl est aussi possible d'installer des écrans de protection phonique provisoires, soit directement autour du pieu à foncer, soit autour de la zone de travail ou de la zone ar protéger pour encager ou dévier les bruits. De simples merlons de terre peuvent suffire.

Mème si les essais de Limelette ne permettent de conclure définitiversent sur le sujet, en vibrofonçage il nous semble important de toujours travailler avec le bon nombre de pinces survant l'axe neutre, comme le recommande le Gujde technique Vibrofoncage (2006) dans le chapitre 2.3.1 a Les pinces pour palplanches 2.

Sur un chantier de vibrofonçage, une grande partie des bruits est générée par le matériel auxiliaire : mâts de guidage, chaînes, élingues, groupes hydrauliques, guides métalliques, moteurs des engins de levage... Ce niveau sonore peut être diminué par des moyens assez simples : utilisation de palplanches pincées, de lubrjfiant dars les griffes libres, de plaques de frottement non métalliques sur les guides (bois, matières synthétiques,...) de groupes hydrauliques biens insonorisés, l'isolation acoustique des élingues, câbles et chaînes de sécurité lenrobage par matériau isolant : mousse, caoutchouc...).

Les constructeurs peuvent aussi essayer de travaller sur le vibrateur luj-même pour l'insonoriser au mieux.

\section{5}

\section{Conclusion}

Les plaintes pour nuisances acoustiques sont nombreuses. Une bonne connaissance des niveaux et des puissances acoustiques des matériels de fonçage par battage et par vibrage est nécessaire pour réduire cette nuisance et rendre le chantier le plus furtif possible. Lanalyse des cing expérimentations en vraie grandeur pendant le fonçage de palplanches, de pieux $\mathrm{H}$ et de pieux en béton armé préfabriqués montre que le battage (marteau et trépideur) est plus bruyant que le vibrofonçage. Suite aux campagnes de La Riche (Boittin, 2002) et de Monchy Saint-Eloi, on peut dire que le fonçage par battage est plus bruyant de 5 à $20 \mathrm{~dB}[\mathrm{~A}$ ] que le fonçage par vibrace.

Pour l'ensemble des mesures, la puissance acoustique pour le battage a varié de $128 \mathrm{~dB}(\mathrm{~A})$ à $130 \mathrm{~dB}(\mathrm{~A})$. La dispersion est faible mais on dispose de peu de mesures. Par ailleurs, la puissance acoustique d'un trépideur [LWA = $128 \mathrm{~dB}(\mathrm{~A}])$ est sensiblement identique à celle des marteawx hydrauliçues (LWA = $130 \mathrm{~dB}(\mathrm{~A})$ ).

La puissance acoustique pour le fonçage par vibrage a varié de $108 \mathrm{~dB}(\mathrm{~A})$ à $126 \mathrm{~dB}(\mathrm{~A})$, soît une différence entre le minimum et le maximum de $18 \mathrm{~dB}(\mathrm{~A})$. Pour mieux maitriser et comprendre cette dispersion de la puissance acoustique, des mesures complémerttaires devront être réalisées sur un maximum de chanLiers avec des vibrateurs et des pieux différents et avec plusieurs sonomètres. Lacquisition de nouveaux sonomètres permettra de mieux exploiter les données er. pouvant distinguer les bruits parasites ponctuels du niveau sonore des engins de chantier.

De pilus, l'expérimentation au Centre scientifique et technique de la construction de Limelette (Belgique) a montré que le fat de positionner les pinces du vibrateur suivant l'axe neutre (Guide technique Vibrofonçage, 2006) a une influence positive sur la diminution du bruit émis pendant le vibrofonçage d'une paire de palplanches. Elle a aussi mis en évidence l'importance de la fréquence et de l'amplitude, sans que l'on puisse vraiment ea tirer une conclusion générale.

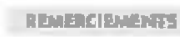

L"aweur remercie particulierment te contre de recherches de ARCE.

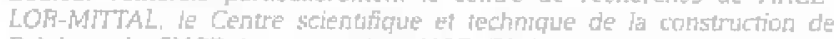
Beigique, la SNCF. les entreprises NGE Gerie Gril et ICE-IHC France

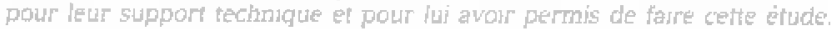

\section{Bibliographie}

Berglund B. Lindvall I. Schwela D. - Guidelines for Community Noise, World Health Organization. "1999.

Boitin R. - DV Construction - Suppression du PN. 186 à la Fiche (37) - Meswre de bruit, Note techrique du LAPC de Blois. 2002

Brüel et Kjaer entreprise - Bruit de l'environnement, 2000.

Circulaire $n^{\circ} 97-110$ du 12 décembre 1997 relative ä la prise en compte du bruit dans la construction de routes nouvelles ou l'aménagrmant de routes extstantes du réseau national.
Guide tachnigue Vibrofongage - Recommandation du projet national Wibrofonçage. Presses des Ponts et Chassées. 2006, $382 \mathrm{p}$.

Mériel B.. Bonhomme B. - Le bruit de chantier. Bullewin des labomatoires des ponts et chaussees - $n^{\circ} 208,199 \bar{r}$, p. 87-98.

NF EN ISO 3744 - Determination des niveaux de puissance acousticue émis par les sources de britt à partír de la pression acoustique.

NF EN 996/A2 - Prescriptions de sécurité. Materiel de battage.
NF $531-110$ - Caractérisation et mesurage des bruits de l'environnement. Gran. deurs fondamentales et méthodes générales d*evaluation.

NF S31-010-Caractérisation et mesurage des bruits de l'environnement. Methodes particulieres de mesurage.

TESPA Technical European Sheet Piling Association - Mise en ceuvre des pal planches en acier. ArcelorMittal, 2007. 